\title{
Effect of Race/Ethnicity and Smoking on Diurnal Variations in Testosterone Levels
}

\author{
Ram B Jain* \\ Centers for Disease Control and Prevention, USA
}

*Corresponding author: Ram B Jain, Private Consultant, Centers for Disease Control and Prevention, 2959 Estate View Court, Dacula, Ga 30019, USA, Tel: 001-910-729-1049, E-mail: jain.ram.b@gmail.com

\begin{abstract}
The effect of race/ethnicity and smoking on diurnal variations in total serum testosterone level (T-TST) was evaluated by analyzing data from NHANES for 2011-2012 for adolescent and adult males. For adolescent males, diurnal variability in T-TST levels was substantially smaller among smokers than nonsmokers for nonHispanic white (NHW) and Hispanics but the reverse was true for non-Hispanic black (NHB). For adult males, morning levels of T-TST were $21-31 \%$ higher than the T-TST levels during evening among NHW and there was no diurnal variation in T-TST levels among NHB nonsmokers but NHB smokers had $3 \%-12 \%$ higher T-TST levels during the morning as compared to the evening. Among adolescents, overall, NHW and NHB had statistically significantly higher T-TST levels in the morning as compared to these levels in the evening $(p \leq 0.02)$. Adult NHW, NHB, and NHAS nonsmokers had statistically significantly lower T-TST levels than NHW, NHB, and NHAS smokers respectively ( $p \leq 0.02$ ).
\end{abstract}

\section{Keywords}

Physical activity, Smoking, Race/ethnicity, Testosterone

\section{Introduction}

In males, testosterone (TST) is the principal male sex hormone and an anabolic steroid. TST plays a key role in the development of male reproductive tissues such as the testis and prostate as well as promoting secondary sexual characteristics such as increased muscle, bone mass, and the growth of body hair [1]. TST is essential for health and wellbeing [2]. The effect of low TST levels on cardiovascular risk factors, Vitamin D levels, and bone mineral density has been extensively studied. TST levels affect body fat distribution and insulin sensitivity in men [3]. Schooling (2013) [4] analyzed National Health and Nutrition Examination (NHANES) III data to report on the relationships between androgen activity and several cardiovascular risk factors. Mondul et al. [5] using data among NHANES III participants did not find TST levels to differ across four quantiles of cholesterol. An inverse relationship between high-density lipoprotein cholesterol and free TST was observed among black and white boys aged 10 to 15 years [6]. The association between Vitamin D and testosterone levels, among others, has been studied by Tak et al. [7], Wulaningsih et al. [8], Lerchbaum et al. [9], Anagnostis et al. [10], Bellastella et al. [11], and Jorde et al. [12]. The effect of TST levels on bone loss has been studied by Barett-Conner et al. [13], Yang et al. [14], Van Hemelrijck et al. [15], and Paller et al. [16]. The association between androgen activity and diabetes has been reported by Selvin et al. [17].
Paller et al. [16] used data from NHANES III and showed low free TST levels to be associated with low bone mineral density. Trabert et al. [18] used NHANES 1999-2004 data and showed an inversed relationship between free and total TST with body fat. Shiels et al. [19] used NHANES III for males and showed association between smoking and higher levels of total TST, number of alcohol drinks and total TST, and a positive relationship between physical activity and free and total TST. It has been postulated that alcohol consumption may damage the Leydig cells or impair the hypothalamus-pituitarygonadal axis [20].

Albertsson-Wikland et al. [20] studied 24-hour nocturnal variations in testosterone levels among boys aged 8.7 to 18.2 years old and concluded that TST levels increase during both day and night in puberty. Mitamura et al. [21] found TST levels to increase during early morning hours among boys aged 4.4 to 19.3 years old. Crofton et al. [22] found increasing levels of TST prior to pubertal onset and into early clinical puberty. Boyar et al. [23] found increased levels of TST during normal nocturnal sleep among 9 pubertal boys but these results were not confirmed among three sexually mature young men. Yong African-American males had 29.4\% higher TST levels at 8 $\mathrm{AM}$ than at $8 \mathrm{PM}$ and Caucasian males had $23.9 \%$ higher TST levels at $8 \mathrm{AM}$ than at $8 \mathrm{PM}$ [24]. In a study of 10 healthy young males (mean age 27.3 years) and 10 healthy elderly males (mean age 70.7 years), mean 24-h levels of non-sex-hormone binding globulin were reported to be $1.91 \pm 0.62 \mathrm{nM} / \mathrm{L}$ among young males and $0.86 \pm 0.01$ $\mathrm{nM} / \mathrm{L}$ among elderly males and circadian rhythm was reported to be blunted with normal aging [25]. Brambilla et al. [26] reported total testosterone levels to be $20-25 \%$ lower at $1600 \mathrm{~h}$ than at $0800 \mathrm{~h}$ among males aged 30-40 years and about $10 \%$ lower at $1600 \mathrm{~h}$ than at $0800 \mathrm{~h}$ among males aged 70 years. The possibility of seasonal variations in TST levels has been reviewed by Smith et al. [27].

Lopez et al. [28] found non-Hispanic black (NHB) 12-15 year adolescent males to have lower total TST levels than non-Hispanic white (NHW) and Mexican Americans (MA) had the higher TST levels as compared to NHB. No racial/ethnic differences were observed between NHW, NHB, and MA among 16-19 year old males for total TST even though MA had the highest levels [28]. No racial/ethnic differences were observed among healthy children aged 6-18 years [29]. In morning session data from NHANES III, NHW and NHB adult males did not differ in TST levels but MA had higher levels than both NHB and NHW [30]. For males aged $\geq 60$ 
years, adjusted levels of TST were highest among Asian-Americans, intermediate among African-Americans, and lowest among whites [31]. TST levels did not differ among African-American and white males without prostate cancer [32]. Among males aged $\geq 24$ years, TST levels did not differ by race after adjustments were made for age, body mass index and waist circumference [33]. In another study which included Singapore-Chinese, African-American, American white, and American Hispanic, and Japanese-American males, TST levels did not differ by race/ethnicity [34]. In a longitudinal study of males aged 30-79 years, [35] also did not find any effect of race/ethnicity on TST levels. Male TST levels did not differ by race/ethnicity among Vietnam era veterans [36]. In a study of college students, black males were found to have $15 \%$ higher TST levels than white males [37].

While diurnal variations among TST levels have been studied in small experimental studies as quoted above, I do not know if diurnal variations in a nationally representative sample of males have been presented before in spite of the fact that NHANES does provide data on serum TST for samples drawn on three different times of the day. In the Mobile Examination Center used in NHANES, participants are assigned to report for examination in one of the three sessions, namely, in the morning, afternoon, and evening. A comparison between the TST levels among these three sets of samples could provide for the magnitude and variability among TST levels by race/ethnicity, smoking, and other factors. Consequently, this study was undertaken with the aim to (i) delineate diurnal variability among total TST levels across three testing sessions, namely, morning, afternoon, and evening, (ii) study the variations in TST levels by age, race/ethnicity, level of physical activity, and smoking status, and (iii) study how age used as a continuous variable, body mass index, waist circumference, fasting time, and alcohol consumption affect TST levels. For 20112012 cycle of NHANES, data on only total TST (T-TST) levels have been released. In the interest of providing the analysis of the most upto-date data, a decision was made to use NHANES 2011-2012 data for males of all ages. It is hypothesized that irrespective of the age, gender, and race/ethnicity, the levels of T-TST will not vary with the time of the testing or the time when blood is drawn.

\section{Materials and Methods}

\section{Data source and study population}

In addition to providing data on androgens for NHANES III (CDC, 2015a) [38] which was conducted between 1988 and 1994, data on the levels of androgens were also publically made available for NHANES cycles 1999-2000 (CDC, 2015b), 2001-2002 (CDC, 2015c) [39,40], and 2003-2004 (CDC, 2015d) [41]. However, these data for NHANES cycles 1999-2000, 2001-2002, and 2003-2004 for sex hormones were made available from surplus samples only or only for those NHANES participants for whom serum samples in sufficient volume were still available after all other analyses were completed. Sampling weights for these data were not available and as such these data cannot be used for making generalizations about U.S. population. Consequently, these data for 1999-2004 were not analyzed for this study. No further data on androgen levels were released until 2011-2012 cycle of NHANES (CDC, 2015e) [42].

Thus, publicly available data from NHANES (CDC, 2015f) [43] for 2011-2012 from demographic, testosterone, body measure, serum cotinine, and physical activity questionnaires were downloaded and match merged. The sampling plan for NHANES was a complex, stratified, multistage, probability cluster designed to be representative of the civilian, non-institutionalized U.S. population. Sampling weights were created in NHANES to account for the complex survey design, including oversampling, survey non-response, and poststratification.

After deleting those for whom T-TST levels were not available, a total of 456 male children aged 6-11 years, 554 adolescents males aged 12-19 years, and 2409 adult males aged $\geq 20$ years were available for analysis. Detailed weighted and un-weighted sample sizes are given in table 1.

\section{Outcome variable}

Because of the highly skewed distribution for T-TST, $\log 10$ transformed levels of T-TST were used as outcome variable in each of the two regression models fitted for adolescents males aged 12-19 years, and adult males aged $\geq 20$ years.

\section{Covariates}

Categorical variables used as covariates in the regression models were: race/ethnicity (non-Hispanic white or NHW, non-Hispanic black or NHB, Hispanics or HISP, and non-Hispanic Asians or NHAS, other unclassified race/ethnicities or OTH), smoking status (nonsmoker, smoker), physical activity level (vigorous, moderate, or neither), and testing session (morning, afternoon, evening). Smokers were defined as those having serum cotinine levels of $\geq 10 \mathrm{ng} / \mathrm{mL}$ and nonsmokers were defined as those having serum cotinine levels of $<10 \mathrm{ng} / \mathrm{mL}$. Continuous variables used as independent variables/ covariates were: age, body mass index, waist circumference, alcohol consumption in grams, and fasting time in hours. A consideration was given to use alcohol consumption as an independent variable. In some of the previous NHANES studies [19], data from food frequency questionnaire available in NHANES were used. However, food frequency questionnaire data were not available for NHANES 2011-2012. Instead, data on total alcohol consumption in grams were available from the two total food frequency questionnaires (CDC,

Table 1: Sample sizes by age, race/ethnicity, and blood draw session. Data from National Health and Nutrition Examination Survey $2011-2012$.

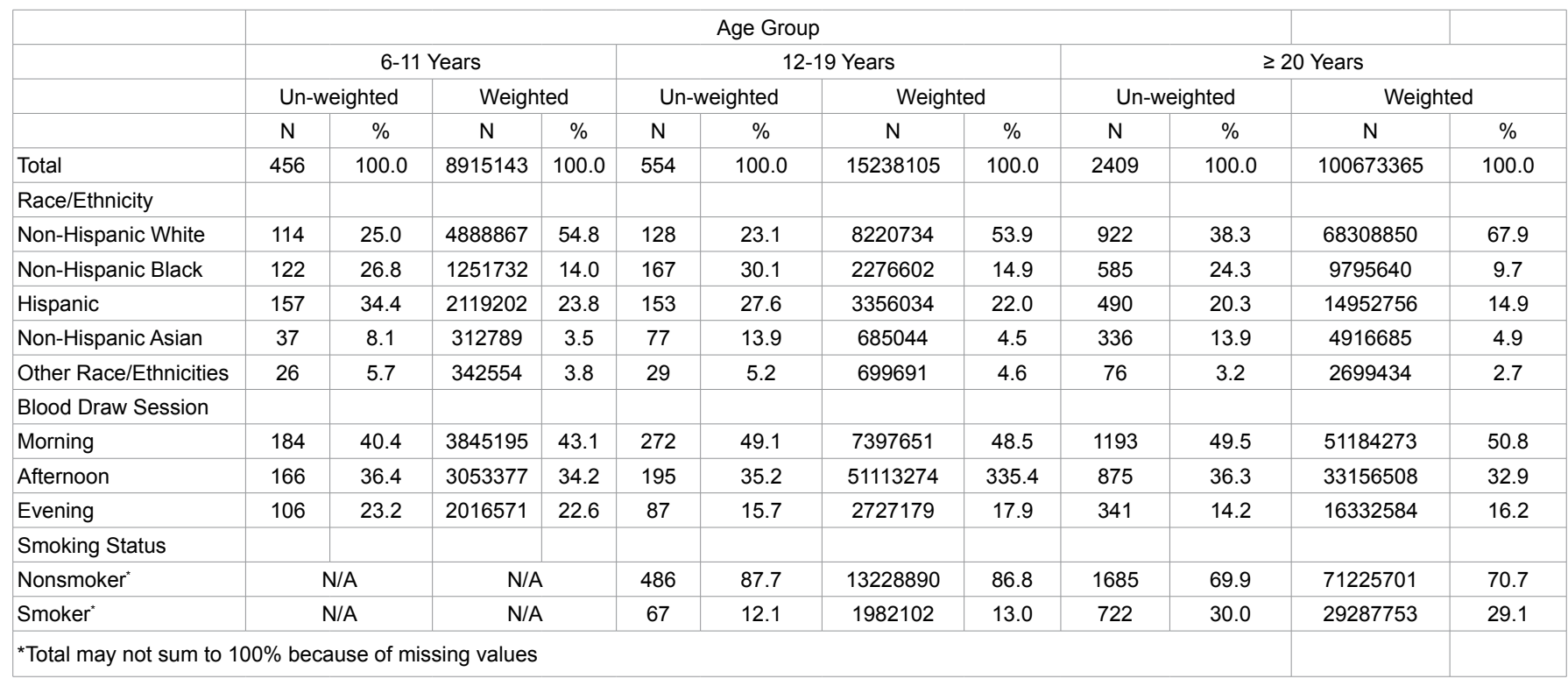


2015g, 2015h) [44,45] and they were used in the analyses. However there were only 38 adolescents who reported consuming $>0$ grams of alcohol. The numbers of person using alcohol was not considered large enough to use alcohol as a variable for adolescents and as such these 38 persons were deleted from the database used for adolescents for this study. Possible biological mechanism between the association of alcohol abuse and plasma testosterone has been provided by [46].

\section{Statistical analysis}

SAS version 9.3 was used to do all statistical analyses. All analyses incorporated appropriate sampling weights and sampling design characteristics. SAS Proc SURVEYMEANS was used to do all univariate analyses including computing unadjusted geometric means by age, race/ethnicity, testing session, and smoking status. Multivariate regression analyses were done using SAS Proc SURVEYREG. Log10 transformed values of T-TST were used as dependent variables in the regression models. Separate regression models were fitted for adolescents and adults. Categorical and continuous variables were used as defined above. Two way interactions between race/ethnicity, smoking status, testing session, and physical activity levels were assessed but were included in the final models only if they were found to be statistically significant at $\alpha=0.05$.

\section{Results}

Based on the un-weighted data, about $25 \%$ of children and adolescents were NHW. Among adults, 38.3\% were NHW (Table 1). Approximately half of the children, adolescents, and adults were tested in the morning session. Only about $15 \%$ the adolescents and adults were tested in the evening session. Among adolescents, $12.1 \%$ were smokers and among adults, $30 \%$ were smokers (Table 1 ).

\section{Effect of race/ethnicity on diurnal variations in T-TST levels based on unadjusted data}

Irrespective of age, T-TST levels during morning session were statistically significantly higher than during the afternoon session and/or the evening session (Table $2, \mathrm{p} \leq 0.01$ ) for the total population. The same was true for NHW (Table 2, $\mathrm{p} \leq 0.05$ ). While for NHB children aged 6-11 years old, T-TST levels for the morning session were statistically significantly higher than for the evening session (Table 2, p < 0.03), no statistically significant differences were found for T-TST levels between morning, afternoon, and evening sessions for adolescents and adults. Neither HISP nor NHAS children nor adolescents had any statistically significant different T-TST levels between morning, afternoon, and evening sessions. However, both HISP and NHAS adult males aged $\geq 20$ years had statistically significantly higher T-TST levels for the morning session than both afternoon and evening sessions (Table $2, \mathrm{p} \leq 0.01$ ). The only instance where T-TST levels were statistically significantly higher in the afternoon session than in the evening session was for all children (Table 2, $\mathrm{p}=0.01$ ) or for NHW children (Table 2, p = 0.03).

\section{Effect of smoking on diurnal variations in T-TST levels based on unadjusted data}

Among nonsmoking male adolescents, except for NHB, T-TST levels were statistically significantly higher for the morning session than the afternoon and/or the evening sessions (Table $3, \mathrm{p} \leq 0.01$ ). There were no statistically significant differences for T-TST levels by testing session among nonsmoking NHB male adolescents. While no statistically significant differences were observed for T-TST levels by testing session for NHW, HISP, and NHAS adolescent smokers, for NHB T-TST levels by testing session were found to be in the order afternoon $>$ morning $>$ evening (Table $3, \mathrm{p} \leq 0.02$ ).

For nonsmoking adults aged $\geq 20$ years, T-TST levels were found to be statistically significantly higher (Table $3, p \leq 0.03$ ) during the morning session than afternoon and/or evening session for the total population, NHW, HISP, and NHAS but no statistically significant differences were found for NHB nonsmoking adults. For adult smokers aged $\geq 20$ years, T-TST levels were found to be statistically significantly higher (Table $3, \mathrm{p} \leq 0.04$ ) during the morning session than afternoon and/or evening session for total population, NHW, $\mathrm{NHB}$, and NHAS but no statistically significant differences were found for HISP adult smoker.

\section{Variability in total T-TST levels among adolescent males based on adjusted data analysis}

Sample size for the model fitted for adolescents was 498 and $\mathrm{R}^{2}$

Table 2: Unadjusted geometric means with $95 \%$ confidence interval in ng/dL by blood draw session for males by age and race/ethnicity. Data from National Health and Nutrition Examination Survey 2011-2012.

\begin{tabular}{|c|c|c|c|c|}
\hline & \multicolumn{3}{|c|}{ Blood Draw Session } & \multirow[t]{2}{*}{ Statistically Significant Differences } \\
\hline & Morning (M) & Afternoon (A) & Evening (E) & \\
\hline \multicolumn{5}{|l|}{ Age: 6-11 Years old } \\
\hline Total & $4.5(3.4-5.9)$ & $3.9(3.2-4.8)$ & $2.5(1.9-3.2)$ & $M>E(p \leq 0.01), A>E(p=0.01)$ \\
\hline Non-Hispanic White & $4.4(3-6.6)$ & $4.2(2.8-6.3)$ & $2.6(1.8-3.7)$ & $M>E(p=0.046), A>E(p=0.034)$ \\
\hline Non-Hispanic Black & $4.5(2.7-7.4)$ & $3.3(2-5.4)$ & $2.1(1.4-3.3)$ & $M>E(p=0.026)$ \\
\hline Hispanic & $4.5(2.7-7.3)$ & $3.5(2.7-4.6)$ & $2.6(1.8-3.8)$ & \\
\hline Other Race/Ethnicities & $4(2.3-6.9)$ & $6.9(2-23.4)$ & $1.1(0.6-1.8)$ & $M>E(p<0.01), A>E(p=0.01)$ \\
\hline \multicolumn{5}{|l|}{ Age: $12-19$ Years old } \\
\hline Total & $336.7(292.4-387.7)$ & $214(161.5-283.6)$ & $180.3(118-275.4)$ & $M>A(p<0.01), M>E(p<0.01)$ \\
\hline Non-Hispanic White & $335.8(261.1-431.9)$ & $179.6(99.2-325)$ & $154.8(77.4-309.3)$ & $M>A(p=0.03)$ \\
\hline Non-Hispanic Black & $363.7(316.9-417.4)$ & $277.5(184.7-416.9)$ & $265.1(194.8-360.6)$ & \\
\hline Hispanic & $326.4(249-427.9)$ & $241.3(200.9-289.8)$ & $228.7(166.5-314.1)$ & \\
\hline Non-Hispanic Asian & $379.1(287.4-500.1)$ & $247.1(168.5-362.4)$ & $260.2(155.1-436.6)$ & \\
\hline \multicolumn{5}{|l|}{ Age: $\geq 20$ Years old } \\
\hline Total & $391.3(373.7-409.6)$ & $347.5(327.1-369.1)$ & $323.5(297-352.5)$ & $M>A(p<0.01), M>E(p<0.01)$ \\
\hline Non-Hispanic White & $387.7(366.6-410)$ & 346 (320.9-373) & $315.6(285.9-348.3)$ & $M>A(p=0.01), M>E(p<0.01)$ \\
\hline Non-Hispanic Black & $374.1(339.2-412.6)$ & $364.7(348.9-381.3)$ & $370(313.8-436.2)$ & \\
\hline Hispanic & $406.4(372.7-443.2)$ & $337.3(317.6-358.1)$ & $336.1(299.8-376.9)$ & $M>A(p<0.01), M>E(p=0.01)$ \\
\hline Non-Hispanic Asian & $415.5(388.5-444.4)$ & $343.2(319.9-368.2)$ & $352(314.5-393.9)$ & $M>A(p<0.01), M>E(p<0.01)$ \\
\hline Other Race/Ethnicities & $411.2(358.4-471.9)$ & $359.4(260.5-496)$ & $288.7(209.8-397.4)$ & $M>E(p=0.046)$ \\
\hline
\end{tabular}


Table 3: Unadjusted geometric means with $95 \%$ confidence interval in ng/dL by blood draw session for males by age, smoking status, and race/ethnicity. Data from National Health and Nutrition Examination Survey 2011-2012.

\begin{tabular}{|c|c|c|c|c|c|}
\hline \multirow[b]{2}{*}{ Smoking Status } & & \multicolumn{3}{|c|}{ Blood Draw Session } & \multirow[t]{2}{*}{ Statistically Significant Differences } \\
\hline & & Morning (M) & Afternoon (A) & Evening (E) & \\
\hline \multirow[t]{6}{*}{ Nonsmokers } & Age: $12-19$ Years old & & & & \\
\hline & Total & $313.5(269.4-364.9)$ & $195.5(143.8-265.9)$ & $167.5(107.1-262)$ & $M>A(p<0.01), M>E(p<0.01)$ \\
\hline & Non-Hispanic White & $303.1(226.2-406.1)$ & $155.4(80-301.9)$ & $139.5(69.8-278.9)$ & $M>E(p<0.01)$ \\
\hline & Non-Hispanic Black & $338.1(285.9-399.9)$ & $260.5(177.1-383.2)$ & $269.6(192.3-377.8)$ & \\
\hline & Hispanic & $323.6(246.6-424.6)$ & $232.6(191.2-282.9)$ & $213.2(138.7-327.8)$ & $M>E(p=0.055)$ \\
\hline & Other Race/Ethnicities & $203.8(133.6-311.1)$ & $279.1(187.6-415.4)$ & $143.6(84.6-243.6)$ & \\
\hline \multirow[t]{6}{*}{ Smokers } & Age: $12-19$ Years old & & & & \\
\hline & Total & $492(421.8-573.9)$ & $440(327.7-590.8)$ & $341.3(226.3-514.8)$ & \\
\hline & Non-Hispanic White & $508.1(417.7-618.1)$ & $499.3(344.1-724.5)$ & $330.9(169.6-645.8)$ & \\
\hline & Non-Hispanic Black & $499.5(434.8-573.9)$ & $743.7(679.8-813.6)$ & $247.1(150.5-405.7)$ & $A>M>E(p<=0.02)$ \\
\hline & Hispanic & $397.8(140.6-1125.7)$ & $273.1(161.9-460.8)$ & $553.3(374.7-817)$ & \\
\hline & Non-Hispanic Asian & $209.4(2.9-15141.3)$ & $365.8(365.8-365.8)$ & No data & \\
\hline \multirow[t]{7}{*}{ Nonsmokers } & Age: $\geq 20$ Years old & & & & \\
\hline & Total & $382(360.9-404.3)$ & $325.7(301.1-352.2)$ & $317(288.2-348.6)$ & $M>A(p<0.01), M>E(p<0.01)$ \\
\hline & Non-Hispanic White & $383.5(356.5-412.5)$ & $321.2(290-355.8)$ & $313.3(281.8-348.5)$ & $M>A(p<0.01), M>E(p<0.01)$ \\
\hline & Non-Hispanic Black & $323(280.8-371.4)$ & $342.7(317.4-370.1)$ & $344(282.1-419.5)$ & \\
\hline & Hispanic & $393.5(368.8-419.8)$ & $327(302.5-353.5)$ & $324.5(275.7-381.8)$ & $M>A(p<0.01), M>E(p=0.03)$ \\
\hline & Non-Hispanic Asian & $402.4(378.5-427.8)$ & $339.3(314.4-366.2)$ & $332.2(297-371.6)$ & $M>A(p<0.01), M>E(p<0.01)$ \\
\hline & Other Race/Ethnicities & $378(317.4-450)$ & $322.2(192.6-539.3)$ & $284.7(172.9-468.8)$ & \\
\hline \multirow[t]{6}{*}{ Smokers } & Age: $\geq 20$ Years old & & & & \\
\hline & Total & $412.7(392.7-433.6)$ & $408.7(385.1-433.7)$ & $343.1(299.4-393.1)$ & $M>E(p<0.01), A>E(p=0.03)$ \\
\hline & Non-Hispanic White & $396.7(375-419.8)$ & $415.3(380.8-453)$ & $323.4(264.6-395.4)$ & $M>E(p=0.03), A>E(p=0.02)$ \\
\hline & Non-Hispanic Black & $457.5(422.4-495.4)$ & $403.8(352.9-462.1)$ & $407.8(339.3-490)$ & $M>A(p=0.04)$ \\
\hline & Hispanic & $458.5(360.3-583.4)$ & $379.8(320.4-450.3)$ & $370.8(314.2-437.6)$ & \\
\hline & Non-Hispanic Asian & $490.5(430.2-559.2)$ & $364(300.4-441.2)$ & $406.2(316.3-521.6)$ & $M>E(p<0.01)$ \\
\hline
\end{tabular}

Table 4: Regression coefficients with p-values for continuous variables used in the model for log 10 transformed values of total serum testosterone Data from National Health and Nutrition Examination Survey 2011-2012.

\begin{tabular}{|c|c|c|c|c|}
\hline \multirow[b]{2}{*}{ Variable } & \multicolumn{2}{|c|}{ Age: $12-19$ Years } & \multicolumn{2}{|c|}{ Age: $\geq 20$ Years } \\
\hline & Slope & P-value & Slope & P-value \\
\hline Age & 0.10979562 & $<0.01$ & -0.001028629 & 0.047 \\
\hline Waist Circumference & -0.007102172 & 0.28 & -0.002863613 & 0.07 \\
\hline Fasting Time in hours & -0.006733424 & 0.65 & 0.004088971 & $<0.01$ \\
\hline Alcohol Consumption in grams & \multicolumn{2}{|c|}{ Not in Model } & 0.000115362 & 0.56 \\
\hline $\mathrm{R}^{2}$ & $40.0 \%$ & & \multicolumn{2}{|c|}{$18.2 \%$} \\
\hline $\mathrm{N}$ & \multicolumn{2}{|c|}{498} & \multicolumn{2}{|c|}{2156} \\
\hline
\end{tabular}

was $40 \%$ (Table 4). Interaction between race/ethnicity and testing session was found to be statistically significant $(p<0.01)$. Age was positively associated with T-TST levels $(\beta=0.1098, p<0.01)$. Body mass index, waist circumference, and fasting time did not affect the levels of T-TST (Table 4). Adjusted T-TST levels for NHW were statistically significantly smaller than for HISP (203.6 vs. $283.2 \mathrm{ng} /$ $\mathrm{dL}, \mathrm{p}=0.03$, Table 5) and NHAS (203.6 vs. $318.8 \mathrm{ng} / \mathrm{dL}, \mathrm{p}<0.01$, Table 5). Physical activity level was not found to be associated with the levels of T-TST (Table 5). T-TST levels were statistically significantly higher during the morning session than during the evening session ( 338.5 vs. $200.3 \mathrm{ng} / \mathrm{dL}, \mathrm{p}=0.02$, Table 5). However, when interaction between race/ethnicity and testing session was taken into account, only NHW (348.8 vs. $140.8 \mathrm{ng} / \mathrm{dL}, \mathrm{p}=0.02$, Table 6$)$ and NHB (382.0 vs. $196.5 \mathrm{ng} / \mathrm{dL}, \mathrm{p}=0.01$, Table 6) had statistically significantly higher adjusted T-TST levels during the morning session as compared to the evening session (Table 6). For NHAS and HISP, statistically significant differences for T-TST levels by testing sessions were not observed (Table 6).

\section{Variability in total T-TST levels among adult males based on adjusted analysis}

Sample size for the model fitted for adolescents was 2156 and $\mathrm{R}^{2}$ was $18.2 \%$ (Table 4 ). Statistically significant differences between race/ethnicity and testing session $(\mathrm{p}<0.01)$ and race/ethnicity and smoking status were observed $(\mathrm{p}=0.03)$. Age was negatively associated with T-TST levels $(\beta=-0.0010, \mathrm{p}=0.047)$. Body mass index and waist circumference were not found to be associated with T-TST levels. Alcohol consumption did not affect the levels of T-TST (Table 4). Fasting time was positively associated with the adjusted levels of T-TST $(\beta=0.0041, \mathrm{p}<0.01$, Table 4$)$.

NHW had statistically significantly lower adjusted levels of T-TST than NHB (361.0 vs. $382.5 \mathrm{ng} / \mathrm{dL}, \mathrm{p}=0.02$, Table 5) and NHB had higher adjusted T-TST levels than NHAS (382.5 vs. $341.9 \mathrm{ng} / \mathrm{dL}, \mathrm{p}=$ 0.02 , Table 5). Smokers were found to have statistically significantly higher levels of T-TST than nonsmokers (384.8 vs. $346.8 \mathrm{ng} / \mathrm{dL}$, Table $5, \mathrm{p}<0.01$ ). Level of physical activity and session during which T-TST serum samples were drawn did not affect the levels of T-TST (Table 5). However, when interaction between race/ethnicity and testing session was considered, morning and afternoon T-TST adjusted levels for NHW were found to be statistically significantly higher than the evening session levels ( 386.0 and 369.9 vs. $329.5 \mathrm{ng} / \mathrm{dL}, \mathrm{p} \leq 0.02$, Table 6).

\section{Diurnal variability among adolescent males}

For the purpose of this study, diurnal variability was defined as 
Table 5: Adjusted geometric means (AGM) with 95\% confidence intervals for serum total testosterone in ng/dL by age, race/ethnicity, smoking stats, and physical activity status. Data from National Health and Nutrition Examination Survey 2011-2012.

\begin{tabular}{|c|c|c|c|c|}
\hline & \multicolumn{2}{|c|}{ Age: $12-19$ Years } & \multicolumn{2}{|c|}{ Age: $\geq 20$ Years } \\
\hline & AGM $(95 \% \mathrm{Cl})$ & $\begin{array}{c}\text { Statistically Significantly } \\
\text { Differences }\end{array}$ & AGM $(95 \% \mathrm{Cl})$ & $\begin{array}{c}\text { Statistically Significantly } \\
\text { Differences }\end{array}$ \\
\hline \multicolumn{5}{|l|}{ Race/Ethnicity } \\
\hline Non-Hispanic White (NHW) & $203.6(151.1-274.5)$ & $\begin{array}{c}\text { NHW }<\text { HISP }(p=0.03), N H W< \\
\text { NHAS }(p<0.01)\end{array}$ & $361(341.7-381.3)$ & $\mathrm{NHW}<\mathrm{NHB}(p=0.04)$ \\
\hline Non-Hispanic Black (NHB) & $267.7(223.1-321.4)$ & & $382.5(363.5-402.5)$ & NHB $>$ NHAS $(p=0.02)$ \\
\hline Hispanic (HSP) & $283.2(219.5-365.6)$ & & $371.7(344.2-401.4)$ & \\
\hline Others (OTH) & $214.2(158.1-290.1)$ & & $370.7(286.6-479.6)$ & \\
\hline \multicolumn{5}{|l|}{ Smoking Status } \\
\hline Nonsmoker (NSM) & $235.9(199.5-279)$ & & $346.8(321.5-374)$ & $N S M<S M(p<0.01)$ \\
\hline Smoker (SM) & $273.2(216-345.4)$ & & $384.8(363.5-407.4)$ & \\
\hline \multicolumn{5}{|l|}{ Physical Activity } \\
\hline Vigorous (V) & $249(181.6-341.4)$ & & $379.2(357-402.7)$ & \\
\hline Neither $(\mathrm{N})$ & $270(237.9-306.5)$ & & $375.1(354-397.4)$ & \\
\hline \multicolumn{5}{|l|}{ Testing Session } \\
\hline Morning (M) & $338.5(270.7-423.4)$ & $M>E(p=0.02)$ & $378.4(355.3-403.1)$ & \\
\hline Afternoon (A) & $241.3(172.1-338.3)$ & & $369(334.7-406.7)$ & \\
\hline Evening (E) & $200.3(142.5-281.4)$ & & $349.1(328.5-371)$ & \\
\hline
\end{tabular}

Table 6: Adjusted geometric means (AGM) with $95 \%$ confidence intervals in ng/dL for total testosterone by race/ethnicity by testing session for those aged $12-19$ and $\geq 20$ years and by race/ethnicity by smoking status or those aged $>=20$ years. Data from National Health and Nutrition Examination Survey $2011-2012$.

\begin{tabular}{|c|c|c|c|c|}
\hline \multirow[b]{2}{*}{ Demographic Group } & \multicolumn{2}{|c|}{ Age 12-19 Years } & \multicolumn{2}{|r|}{ Age $>=20$ Years } \\
\hline & $\operatorname{AGM}(95 \% \mathrm{Cl})$ & $\begin{array}{l}\text { Statistically Significantly } \\
\text { Differences }\end{array}$ & $\operatorname{AGM}(95 \% \mathrm{Cl})$ & Statistically Significantly Differences \\
\hline Non-Hispanic White Morning Session (NHW_M) & $348.8(233.8-520.3)$ & NHW_M > NHW_E $(p=0.02)$ & $386(361.5-412.2)$ & NHW_M > NHW_E $(p<0.01)$ \\
\hline Non-Hispanic White Afternoon Session (NHW_A) & $171.9(84.3-350.7)$ & NHW_A $<$ NHAS_A $(p<0.01)$ & $369.9(343.8-398)$ & NHW_A > NHW_E $(p=0.02)$ \\
\hline Non-Hispanic White Evening Session (NHW_E) & $140.8(86.1-230.2)$ & & $329.5(298.9-363.1)$ & NHW_E $<$ NHB_E $(p=0.02)$ \\
\hline Non-Hispanic Black Morning Session (NHB_M) & $382(297.4-490.7)$ & $\begin{array}{l}\text { NHB_M }>\text { NHB_E }(p=0.01) \\
\text { NHB_M }>\text { OTH_M }(p=0.02)\end{array}$ & $374.5(342.3-409.7)$ & \\
\hline Non-Hispanic Black Afternoon Session (NHB_A) & $255.7(187.6-348.5)$ & & $387.2(372.3-402.7)$ & NHB_A $>$ NHAS_A $(p<0.01)$ \\
\hline Non-Hispanic Black Evening Session (NHB_E) & $196.5(123.3-313.1)$ & & $386(342.1-435.7)$ & \\
\hline Hispanics Morning Session (HISP_M) & $379.9(250.4-576.4)$ & & $393.5(360.1-429.9)$ & HISP_M > NHAS_M $(p=0.02)$ \\
\hline Hispanics Afternoon Session (HISP_A) & $264.1(186.3-374.3)$ & & $379.8(353.4-408.3)$ & HISP_A > NHAS_A $(p=0.02)$ \\
\hline Hispanics Evening Session (HISP_E) & $226.5(137.6-372.8)$ & & $343.5(296.9-397.3)$ & \\
\hline Non-Hispanic Asian Morning Session (NHAS_M) & $464.9(283.9-761.5)$ & $\begin{array}{l}\text { NHAS_M }>\text { NHAS_A }(p=0.04), \\
\text { NHAS_M }>\text { OTH_M }(p=0.02)\end{array}$ & $353.3(323.3-386)$ & \\
\hline Non-Hispanic Asian Evening Session (NHAS_E) & $280.7(188.7-417.5)$ & NHAS_E > OTH_E $(p=0.01)$ & $342.8(301.6-389.6)$ & \\
\hline Other Race/Ethnicities Morning Session (OTH_M) & $188.9(129-276.7)$ & & $386.3(292.8-509.8)$ & \\
\hline Other Race/Ethnicities Afternoon Session (OTH_A) & $283.8(168.6-477.6)$ & OTH_A > OTH_E $(p=0.047)$ & $381(237.2-611.9)$ & \\
\hline Other Race/Ethnicities Evening Session (OTH_E) & $183.2(123.3-272.2)$ & & $346.2(283.5-422.8)$ & \\
\hline Non-Hispanic White Nonsmokers (NHW_NSM) & & & $351.3(332.1-371.7)$ & NHW_NSM < NHW_SM $(p=0.03)$ \\
\hline Non-Hispanic White Smokers (NHW_SM) & & & $370.9(348.1-395.3)$ & \\
\hline Non-Hispanic Black Nonsmokers (NHB_NSM) & & & $363(339.2-388.6)$ & $\begin{array}{l}\text { NHB_NSM }<\text { NHB_SM }(p=0.02), \\
\text { NHB_NSM }>\text { NHAS_NSM }(p=0.01)\end{array}$ \\
\hline Non-Hispanic Black Smokers (NHB_SM) & & & $403(378.1-429.7)$ & \\
\hline Hispanics Nonsmokers (HISP_NSM) & & & $354.8(336-374.6)$ & HISP_NSM > NHAS_NSM $(p=0.02)$ \\
\hline Hispanics Smokers (HISP_SM) & & & $389.3(343.8-440.9)$ & \\
\hline Non-Hispanic Asian Nonsmokers (NHAS_NSM) & & & $319.9(298.9-342.5)$ & NHAS_NSM $<$ NHAS_SM $(p=0.02)$ \\
\hline Non-Hispanic Asian Smokers (NHAS_SM) & & & $365.3(326.9-408.1)$ & \\
\hline Other Race/Ethnicity Nonsmokers (OTH_NSM) & & & $346.4(251.6-477)$ & \\
\hline
\end{tabular}

the ratio of the geometric mean for T-TST for the morning session divided by the geometric mean for T-TST for the evening session. Ratios of both unadjusted (RU) as well as adjusted geometric means (RA) were computed and are given in table 7. While RA and RU for adolescent nonsmokers were similar or 1.87 and 1.85 respectively, RU for smokers was 1.44 and RA for smokers was 1.84 . Consequently, morning T-TST levels may be more than $80 \%$ higher than evening T-TST levels. For nonsmoker adolescents, morning T-TST levels for NHW may be double of what they are for the evening session $(\mathrm{RU}=$ $2.17, \mathrm{RA}=2.06$, Table 7). The same was found to be true for NHW smoker adolescents since RA $=2.02$. For NHB smokers also, morning
T-TST levels could be double of what they are for the evening testing $(\mathrm{RA}=2.02)$ but the morning-evening differences for $\mathrm{NHB}$ nonsmokers were only about $25 \%$ (Table 7 ). Similar patterns were observed for NHAS smokers and nonsmokers.

\section{Diurnal variability among adult males}

Smoking did not affect diurnal variability to the same degree for adults than it did for adolescents irrespective of race/ethnicity since both RA and RU were about 1.2. Winters et al. [24] found young Caucasians to have $23.9 \%$ higher TST levels at $8 \mathrm{AM}$ as compared to the levels at 8 PM. In this study, NHW were found to have $20-23 \%$ higher T-TST 
Table 7: The ratios of the unadjusted geometric means (RU) and adjusted geometric means (RA) for total TST levels for morning to evening session by age, race/ ethnicity, and smoking status. Data from National Health and Nutrition Examination Survey 2011-2012.

\begin{tabular}{|c|c|c|c|c|c|c|}
\hline \multirow{2}{*}{$\begin{array}{l}\text { Smoking Status } \\
\text { Nonsmokers }\end{array}$} & \multirow[b]{2}{*}{ Age: $12-19$ Years old } & \multicolumn{3}{|c|}{$\mathrm{N}$} & \multirow[b]{2}{*}{$\mathrm{RU}$} & \multirow[b]{2}{*}{$\mathrm{RA}$} \\
\hline & & Morning & Afternoon & Evening & & \\
\hline & Total & 232 & 117 & 77 & 1.87 & 1.85 \\
\hline & Non-Hispanic White & 52 & 33 & 18 & 2.17 & 2.06 \\
\hline & Non-Hispanic Black & 77 & 53 & 13 & 1.25 & 1.34 \\
\hline & Hispanic & 63 & 54 & 25 & 1.52 & 1.45 \\
\hline & Non-Hispanic Asian & 32 & 26 & 15 & 1.54 & 1.3 \\
\hline & Other Race/Ethnicities & 8 & 11 & 6 & 1.42 & 2.14 \\
\hline \multirow[t]{7}{*}{ Smokers } & Age: $12-19$ Years old & & & & & \\
\hline & Total & 40 & 17 & 10 & 1.44 & 1.84 \\
\hline & Non-Hispanic White & 14 & 7 & 4 & 1.54 & 2.02 \\
\hline & Non-Hispanic Black & 18 & 3 & 3 & 2.02 & 1.82 \\
\hline & Hispanic & 3 & 5 & 2 & 0.72 & 1.07 \\
\hline & Non-Hispanic Asian & 3 & 1 & 0 & 0.57 & 2.47 \\
\hline & Other Race/Ethnicities & 2 & 1 & 1 & 1.67 & 0.81 \\
\hline \multirow{6}{*}{ Nonsmokers } & Total & 844 & 610 & 231 & 1.21 & 1.2 \\
\hline & Non-Hispanic White & 317 & 204 & 95 & 1.22 & 1.21 \\
\hline & Non-Hispanic Black & 152 & 161 & 47 & 0.94 & 1 \\
\hline & Hispanic & 205 & 125 & 52 & 1.21 & 1.2 \\
\hline & Non-Hispanic Asian & 150 & 98 & 29 & 1.21 & 1.17 \\
\hline & Other Race/Ethnicities & 20 & 22 & 8 & 1.33 & 1.62 \\
\hline \multirow[t]{7}{*}{ Smokers } & Age: $\geq 20$ Years old & & & & & \\
\hline & Total & 348 & 264 & 110 & 1.20 & 1.23 \\
\hline & Non-Hispanic White & 161 & 105 & 39 & 1.23 & 1.31 \\
\hline & Non-Hispanic Black & 94 & 96 & 34 & 1.12 & 1.03 \\
\hline & Hispanic & 56 & 32 & 20 & 1.21 & 1.17 \\
\hline & Non-Hispanic Asian & 27 & 21 & 11 & 1.07 & 1.08 \\
\hline & Other Race/Ethnicities & 10 & 10 & 6 & 1.50 & 1.22 \\
\hline
\end{tabular}

levels during morning session than during the evening session which is in confirmation with the results of Winters et al. [24] meaning NHW do have certain amount of diurnal variability in their T-TST levels.

There was almost no diurnal variation in T-TST levels for NHB nonsmokers (diurnal RU $=0.94, \mathrm{RA}=1.0$ ) but NHB smokers did have about $12 \%$ higher T-TST levels based on RU but only $3 \%$ based on RA during the morning session than during the evening session (Table 7). This means that NHB nonsmokers do not have diurnal variability in their T-TST levels but NHB smokers do exhibit some diurnal variability in their T-TST levels. Thus, finding by Winters et al. [24] that found young African-Americans had 29.4\% higher TST levels at $8 \mathrm{AM}$ as compared to the levels at $8 \mathrm{PM}$ is only partially confirmed and that too among smokers. The differences in the results by the two studies may be due to ages covered by these two studies.

Among NHAS, while diurnal variability ratio for nonsmokers was 1.21 , it was 1.07 among smokers.

\section{Discussion}

Effect of race/ethnicity, smoking, physical activity, testing session and other factors on T-TST levels among male adolescents

Using NHANES III data, Lopez et al. [28] found racial/ethnic differences in the levels of T-TST among 12-15 years old but not so among 16-19 years old. For the combined data for 12-19 years old for this study, NHW were found to have lower adjusted T-TST levels than both HISP and NHAS but not when compared with NHB (Table 5). However, when interaction between race/ethnicity and testing session was taken into account, racial/ethnic differences in T-TST levels almost disappeared (Table 6). However, morning T-TST levels were found to be statistically significantly higher for at least NHW and NHB when compared with evening T-TST levels (Table 6). In fact, morning T-TST levels may be double of what they are during evening testing for both smoker and nonsmoker NHW and $80 \%$ higher for NHB smokers (Table 7). Thus, role of race/ethnicity in determining
T-TST levels among male adolescents seems to be limited but both NHW and NHB adolescents do have diurnal variability in their T-TST levels.

Effect of race/ethnicity, smoking, physical activity, testing session, and other factors on T-TST levels among male adults

The order of T-TST levels by race/ethnicity was NHAS $<$ NHW $<$ NHB. These results are not comparable with the results provided by Rohrmann et al. [30] because while Rohrmann et al. [30] used only morning session data, this study used data for all three sessions in the adjusted models. For this study, NHW were found to have the lowest T-TST levels when compared with NHB and Hispanics as was observed by Wu et al. [31] but Asian-Americans were not found to have the highest levels as was reported by Wu et al. [31] which may be because while $\mathrm{Wu}$ et al. [31] study was done among those aged $\geq$ 60 years, this study was done among all those aged $\geq 20$ years. Also, while Gapstur et al. [33] did not find any racial/ethnic differences among $\geq 24$ years old males, in this study, NHW were found to have lower T-TST levels than NHB.

\section{Limitations of the Study}

This study was based on cross-sectional data and as such, diurnal variations reported in this study need to be interpreted with caution. True diurnal variability can only be determined if TST levels are determined for the same persons over time. There were many pairwise comparisons that were made, for example, between NHW and NHB, NHB and HISP etc. each with its own p-value. The possibility of inflated type I error with so many comparisons should not be overlooked. There are many corrective methods that are available to adjust for multiple comparisons so as to have overall p-value below the nominal level which generally is 0.05 . Some of these corrective methods include Bonferoni correction, Newman-Keuls method, Scheffe method, and Tukey's least significant different method. However, as could be expected, different correction methods may lead to different pair-wise comparisons being labeled as statistically 
significant. Consequently, none of the correction methods were used to generate results for this study. Instead, actual p-values for each pair-wise comparison as well as regression slopes were presented. In the opinion of this author, a decision should be made by the clinicians about which of the possible and how many comparisons are of clinical interest/significance. Once this has been decided, a decision should be made about what is the total level of type I error that can be allowed for "all" comparisons of interest which usually could be 0.05 . Following this, the clinician may want to decide of the total allowable type I error, how much type I error should be allocated to which comparison. Once this allocation has been made, the clinician should look at what is the computed type I error (or p -value) attached with the comparisons of interest. If the computed type I error or p-value for a specific comparison is higher than the allocated type I error, the clinician may want to designate that comparison as "clinically insignificant". If the computed type I error or p-value for a specific comparison is lower than the allocated type I error, the clinician may want to designate that comparison as "clinically significant". The possibility of a result that is "statistically insignificant" being "clinically significant" should also not be ruled out. It should be remembered that there is an inverse association between type I and type II errors. It may be tempting to be too conservative in applying type I error but that also means type II error is inflated accordingly. A clinician may decide what is of greater importance/significance, namely, making a conclusion about the hypothesized differences being statistically/clinically significant when, in fact, they are not (type I error) or making a conclusion about the hypothesized differences not being statistically/clinically significant when, in fact, they are (type II error). Different clinicians may attach different values to type I and type II errors.

\section{Summary and Conclusion}

Among adolescent males, (i) diurnal variability in T-TST levels was substantially smaller among smokers than nonsmokers except for NHB for whom the reverse was true, (ii) the order of diurnal variability for T-TST levels by race/ethnicity was NHW > NHAS > HISP > NHB, (iii) NHW had lower levels of T-TST than HISP and NHAS, (iv) smoking and physical activity did not affect the levels of T-TST, (iv) morning T-TST levels among NHW and NHB were higher than evening T-TST levels, and (v) T-TST levels increased with age.

Among adult males, (i) NHW had 21-31\% higher T-TST levels during morning than evening for both smokers and nonsmokers, (ii) there was no diurnal variation in T-TST levels among NHB nonsmokers but NHB smokers had 3\%-12\% higher T-TST levels during morning as compared to evening, (iii) NHW had higher morning and afternoon T-TST levels than evening T-TST levels, (iv) NHW, NHB, and NHAS smokers had higher T-TST levels than NHW, NHB, and NHAS nonsmokers, respectively (iii) T-TST levels decreased with increase in age.

\section{Declaration of Interest}

The author declares that there is no conflict of interest that could be perceived as prejudicing the impartiality of the research reported.

\section{Funding}

This research did not receive any specific grant from any funding agency in the public, commercial or not-for-profit sector.

\section{References}

1. Mooradian AD, Morley JE, Korenman SG (1987) Biological actions of androgens. Endocr Rev 8: 1-28.

2. Bassil N, Alkaade S, Morley JE (2009) The benefits and risks of testosterone replacement therapy: a review. Ther Clin Risk Manag 5: 427-448.

3. Stahlhut RW, van Wijngaarden E, Dye TD, Cook S, Swan SH (2007) Concentrations of Urinary Phthalate Metabolites Are Associated with Increased Waist Circumference and Insulin Resistance in Adult U.S. Males. Environ Health Perspec 115: 876-882.

4. Schooling CM (2013) Androgen activity, ischaemic heart disease and risk factors among men in NHANES III. Eur J Clin Invest 43: 1273-1281.

5. Mondul AM, Selvin E, Rohrmann S, Menke A, Feinleib M, et al. (2010)
Association of serum cholesterol and cholesterol-lowering drug use with serum sex steroid hormones in men in NHANES III. Cancer Causes Control 21: $1575-1583$

6. Morrison JA, Sprecher DL, Biro FM, Apperson-Hansen C, Dipaola LM (2002) Serum testosterone associates with lower high-density lipoprotein cholesterol in black and white males, 10 to 15 years of age, through lowered apolipoprotein Al and All concentrations. Metabolism 51: 432-437.

7. Tak YJ, Lee JG1, Kim YJ, Park NC, Kim SS, et al. (2015) Serum 25-hydroxyvitamin $D$ levels and testosterone deficiency in middle-aged Korean men: a cross-sectional study. Asian J Androl 17: 324-328.

8. Wulaningsih W, Van Hemelrijck M, Michaelsson K, Kanarek N, Nelson WG, et al. (2014) Association of serum inorganic phosphate with sex steroid hormones and vitamin $D$ in a nationally representative sample of men. Andrology 2: 967-976.

9. Lerchbaum E, Pilz S, Trummer C, Rabe T, Schenk M, et al. (2014) Serum vitamin $\mathrm{D}$ levels and hypogonadism in men. Andrology 2: 748-754.

10. Anagnostis P, Karras S, Goulis DG (2013) Vitamin D in human reproduction: a narrative review. Int J Clin Pract 67: 225-235.

11. Bellastella G, Maiorino MI, Olita L, Capuano A, Rafaniello C, et al. (2014) Vitamin $D$ deficiency in type 2 diabetic patients with hypogonadism. J Sex Med 11: 536-542.

12. Jorde R, Grimnes G, Hutchinson MS, Kjærgaard M, Kamycheva E, et al. (2013) Supplementation with vitamin D does not increase serum testosterone levels in healthy males. Horm Metab Res 45: 675-681.

13. Barrett-Connor E, Laughlin GA, Li H, Nielson CM, Wang PY, et al. (2012) The association of concurrent vitamin $\mathrm{D}$ and sex hormone deficiency with bone loss and fracture risk in older men: the osteoporotic fractures in men (MrOS) study. J Bone Miner Res 27: 2306-2313.

14. Yang B, Sun $H$, Wan $Y$, Wang $H$, Qin $W$, et al. (2012) Associations between testosterone, bone mineral density, vitamin $\mathrm{D}$ and semen quality in fertile and infertile Chinese men. Int J Androl 35: 783-792.

15. Van Hemelrijck M, Michaelsson K, Nelson WG, Kanarek N, Dobs A, et al. (2013) Association of serum calcium with serum sex steroid hormones in men in NHANES III. Aging Male 16: 151-158.

16. Paller CJ, Shiels MS, Rohrmann S, Basaria S, Rifai N, et al. (2009) Relationship of sex steroid hormones with bone mineral density (BMD) in a nationally representative sample of men. Clin Endocrinol (Oxf) 70: 26-34.

17. Selvin E, Feinleib M, Zhang L, Rohrmann S, Rifai N, et al. (2007) Androgens and diabetes in men: results from the Third National Health and Nutrition Examination Survey (NHANES III). Diabetes Care 30: 234-238.

18. Trabert B, Graubard BI, Nyante SJ, Rifai N, Bradwin G, et al. (2012) Relationship of sex steroid hormones with body size and with body composition measured by dual-energy X-ray absorptiometry in US men. Cancer Causes Control 23: 1881-1891.

19. Shiels MS, Rohrmann S, Menke A, Selvin E, Crespo CJ, et al. (2009) Association of cigarette smoking, alcohol consumption, and physical activity with sex steroid hormone levels in US men. Cancer Causes Control 20: 877886 .

20. Albertsson-Wikland K, Rosberg S, Lannering B, Dunkel L, Selstam G, et al. (1999) Twenty-four-hour profiles of luteinizing hormone, follicle-stimulating hormone, testosterone, and estradiol levels: a semilongitudinal study throughout puberty in healthy boys. J Clin Endocrinol Metab 82: 541-549.

21. Mitamura R, Yano K, Suzuki N, Ito Y, Makita Y, et al. (1999) Diurnal rhythms of luteinizing hormone, follicle-stimulating hormone, and testosterone secretion before the onset of male puberty. J Clin Endocrinol Metab 84: 29-37.

22. Crofton PM, Evans AE, Groome NP, Taylor MR, Holland CV, et al. (2002) Inhibin $B$ in boys from birth to adulthood: relationship with age, pubertal stage FSH and testosterone. Clin Endocrinol (Oxf) 56: 215-221.

23. Boyar RM, Rosenfeld RS, Kapen S, Finkelstein JW, Roffwarg HP, et al. (1974) Human puberty. Simultaneous augmented secretion of luteinizing hormone and testosterone during sleep. J Clin Invest 54: 609-618.

24. Winters SJ, Brufsky A, Weissfeld J, Trump DL, Dyky MA, et al. (2001) Testosterone, sex hormone-binding globulin, and body composition in young adult African American and Caucasian men. Metabolism 50: 1242-1247.

25. Plymate SR, Tenover JS, Bremner WJ (1989) Circadian variation in testosterone, sex hormone-binding globulin, and calculated non-sex hormone-binding globulin bound testosterone in healthy young and elderly men. J Androl 10: 366-371.

26. Brambilla DJ, Matsumoto AM, Araujo AB, McKinlay JB (2009) The effect of diurnal variation on clinical measurement of serum testosterone and other sex hormone levels in men. J Clin Endocrinol Metab 94: 907-913.

27. Smith RP, Coward RM, Kovac JR, Lipshultz LI (2013) The evidence for seasonal variations of testosterone in men. Maturitas 74: 208-212.

28. Lopez DS, Peskoe SB, Joshu CE, Dobs A, Feinleib M, et al. (2013) Racial/ 
ethnic differences in serum sex steroid hormone concentrations in US adolescent males. Cancer Causes Control 24: 817-826.

29. Richards RJ, Svec F, Bao W, Srinivasan SR, Berenson GS (1992) Steroid hormones during puberty: racial (black-white) differences in androstenedione and estradiol-the Bogalusa Heart Study. J Clin Endocrinol Metab 75: 624-631.

30. Rohrmann S, Nelson WG, Rifai N, Brown TR, Dobs A, et al. (2007) Serum estrogen, but not testosterone, levels differ between black and white men in a nationally representative sample of Americans. J Clini Endocrinol Metab 92: $2519-2525$

31. Wu AH, Whittemore AS, Kolonel LN, John EM, Gallagher RP, et al (1995) Serum androgens and sex hormone-binding globulins in relation to lifestyle factors in older African-American, white, and Asian men in the United States and Canada. Cancer Epidemiol Biomarkers Prev 4: 735-741.

32. Eastham JA, May RA, Whatley T, Crow A, Venable DD, et al. (1998) Clinica characteristics and biopsy specimen features in African-American and white men without prostate cancer. J Natl Cancer Inst 90: 756-760.

33. Gapstur SM, Gann PH, Kopp P, Colangelo L, Longcope C, et al. (2002) Serum androgen concentrations in young men: a longitudinal analysis of associations with age, obesity, and race. The CARDIA male hormone study. Cancer Epidemiol Biomarkers Prev 11: 1041-1047.

34. Cheng I, Yu MC, Koh WP, Pike MC, Kolonel LN, et al. (2005) Comparison of prostate-specific antigen and hormone levels among men in Singapore and the United States. Cancer Epidemiol Biomarkers Prev 14: 1692-1696.

35. Litman HJ, Bhasin S, Link CL, Araujo AB, McKinlay JB (2006) Serum androgen levels in black, Hispanic, and white men. J Clin Endocrinol Metab 91: 4326-4334.
36. Ellis L, Nyborg H (1992) Racial/ethnic variations in male testosterone levels: a probable contributor to group differences in health. Steroids 57: 72-75

37. Ross R, Bernstein L, Judd H, Hanisch R, Pike M, et al. (1986) Serum testosterone levels in healthy young black and white men. J Natl Cancer Inst 76: 45-48.

38. CDC (2015a) Centers for Disease Control. National Health and Nutrition Examination Survey.

39. CDC (2015b) Centers for Disease Control. National Health and Nutrition Examination Survey.

40. CDC (2015c) Centers for Disease Control. National Health and Nutrition Examination Survey.

41. CDC (2015d) Centers for Disease Control. National Health and Nutrition Examination Survey.

42. CDC (2015e) Centers for Disease Control. National Health and Nutrition Examination Survey.

43. CDC (2015f) Centers for Disease Control. National Health and Nutrition Examination Survey.

44. CDC (2015g) Centers for Disease Control. National Health and Nutrition Examination Survey.

45. CDC (2015h) Centers for Disease Control. National Health and Nutrition Examination Survey.

46. Maneesh M, Dutta S, Chakrabarti A, Vasudevan DM (2006) Alcohol abuseduration dependent decrease in plasma testosterone and antioxidants in males. Indian J Physiol Pharmacol 50: 291-296. 